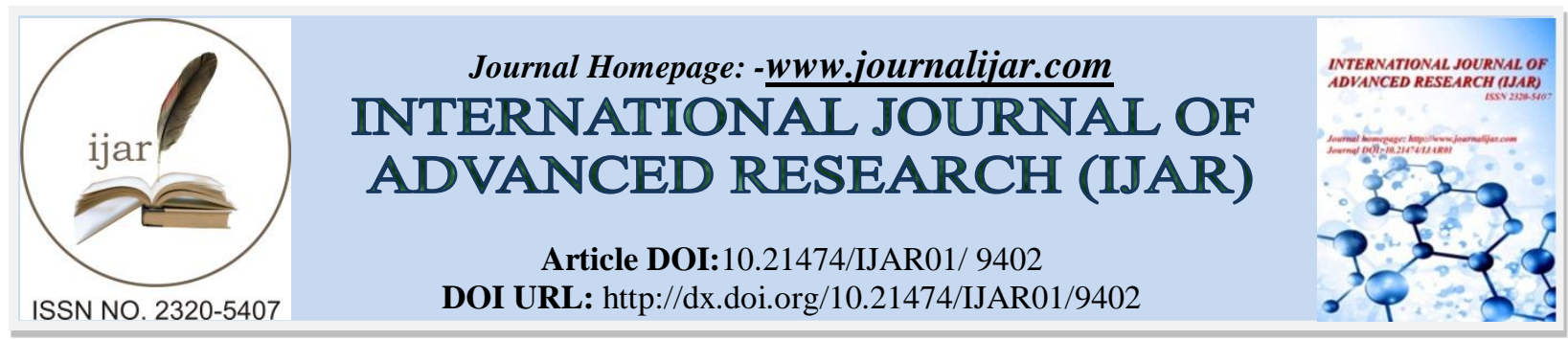

RESEARCH ARTICLE

\title{
SURVEY ON SOLID WASTE MANAGEMENT IN PUBLIC HOSPITALS AND PRIVATE HOSPITALS IN THE CITY OF TANGIER.
}

Y. Ouzekhti Yettefti, S. Ajbar El Gueriri, H. El Cadi, A. Mouhssine And J. Brigui.

Laboratory of Chemical Engineering and Valorization of the resources Faculty of Sciences and Techniques of Tangier, Abdel Malek Essaâdi University, Km10 Route de Ziaten, BP 416 Tangier, Morocco.

\section{Manuscript Info}

Manuscript History

Received: 17 May 2019

Final Accepted: 19 June 2019

Published: July 2019

Key words:-

Medical waste; Waste management;

Hospital waste; Infectious waste.

\begin{abstract}
In Morocco, there is little information available concer ${ }^{1}$ ning the production, composition, handling, and disposal of hospital waste. The purpose of this study is to investigate the Hospital Waste Management in 4 public hospitals, 6 private hospitals for-profit company and a private non-profit organization in the city of Tangier. The results showed that the generation rate per capita of Hospital Waste in Tangier is among $2.12 \mathrm{~kg}$ per bed per day in the public hospitals and on average up to $3.58 \mathrm{~kg}$ in private hospitals. Solid waste of public hospitals consists $74.9 \%$ of household and similar waste and $25.1 \%$ of infectious waste.

In private hospitals, the household and similar waste is $69.8 \%$ and infectious waste is $30.2 \%$ of the total waste produced. This work shows that, for most health facilities studied, general waste has been mixed with infectious waste, and were collected, transported and disposed at the landfill.
\end{abstract}

Copy Right, IJAR, 2019,. All rights reserved.

\section{Introduction:-}

The production rate of waste in developing countries increases with an increase in population [7] .The pollutants present in this waste can affect water resources, air, and soil [9] .

Activities of health care can lead to the production of various types of solid waste which can have harmful effects on human health and plants. Hospital Waste (HW), due to its infectious nature, can be one of the most dangerous pollution sources [12].

Hospitals and medical centers have a special responsibility with regard to the production of waste. They must ensure that's the waste generated result no negative impacts on the environment and public health [4].

According to the definition of the World Organization of the Health [12] solid waste generated during the diagnosis, treatment and vaccination of humans or animals, including biological tests, but not limited to: Bandages, soiled or steeped in blood, culture and other glass utensils, including also the surgical gloves and needles. 
The environmental risks caused by poor management of waste are considered to be one of the biggest problems in developing countries. As the volume and complexity of health care waste increase, the risk of transmission of the disease during the transport and disposal is also growing.

Today, it is identified that specific categories of Medical Waste (MW) are among the waste containing a dangerous potential. Of all waste generated in health centers, 75 to $90 \%$ of the waste is not dangerous or general waste. While 10 to $25 \%$ of waste is still infectious and dangerous and can cause many risks for health.

Although the proportion of infectious and hazardous waste is relatively low, a bad management of this waste can cause contamination of a large volume of general waste [5].

Several studies have shown that the highest risk of transmission of disease in workers in contact with hazardous waste is associated with AIDS, hepatitis B and C and tuberculosis [3].

In developing countries, the importance given to HW is low [14], and in many countries, the transport and disposal of hazardous waste and MW remain associated with HSW [6].

In most Moroccan cities, Household and Similar Waste (HSW)and Health Care Waste (HCW) is collected together for disposal in landfills, waste dumps or poorly designed incinerators, creating operational problems.

Medical Waste Management (MWM) planning is essential to avoid adverse effects on human health and the environment. For a successful implementation of the MW management plan, the availability of sufficient and up-todate information on the quantity and composition of waste produced is crucial [3].

Over the years, several waste management systems, procedures, and methods have been reported for proper handling and disposal of HW, including land filling [8], incineration [15], autoclaving and recycling [10].

In general, there is not just one solution to HW management problems. In most cases, several approaches are combined because each practice has its strengths and weaknesses [12].

In addition, WHO has put in place various guidelines for safe, effective and environmentally sound methods for the handling and disposal of HW.

The objective of this study is to evaluate solid waste management practices in Tangier by studying eleven health care facilities distributed between public and private hospitals. This will determine the quantity, composition, and waste of health care per person, in order to have a better waste management and reduce the risks of hazardous waste.

\section{Materials and methods:- \\ Study area}

The city of Tangier is located in the extreme north-west of Morocco. This area isestimated to be $863 \mathrm{~km}^{2}$ and its population is equivalent to $1,065,601$ inhabitants according to the 2014 census. It is considered as the capital of Tangier-Tetouan-Al-Hoceima region. This field of study includes 11 health care institutions (4 public and 7 private hospitals). 


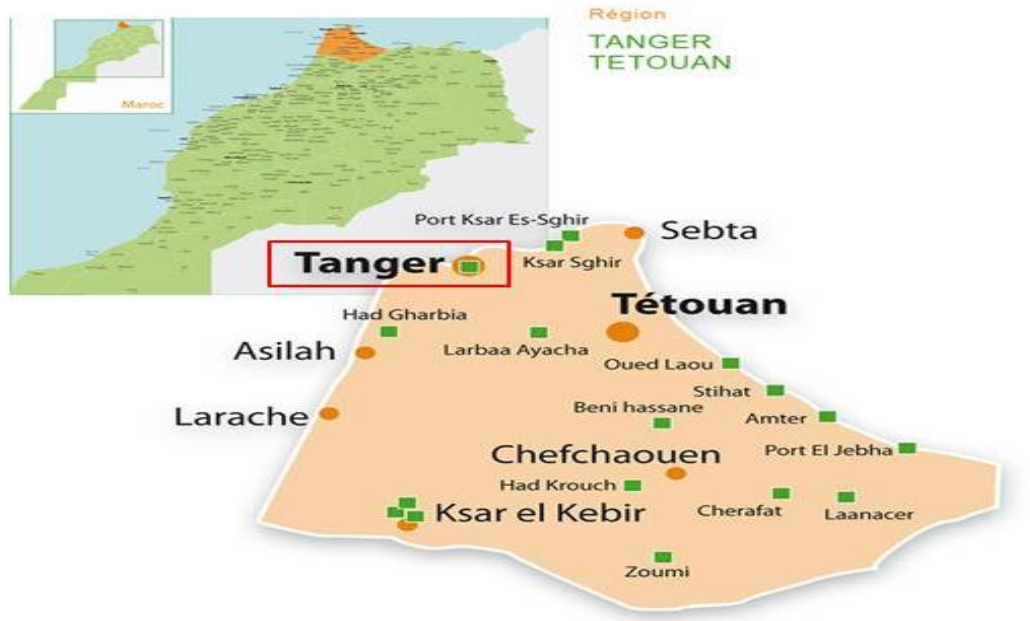

Figure 1:-Map showing the study area (source: design 2012: claw and Strategies)

\section{Methodology:-}

Before starting our investigation into HWM in the city's health institutions, we are interested in studies of the city's history. We realized a lack of accurate data, apart from general national estimates. In an attempt to identify the reality of MWM, we had to look more closely at this issue and we have developed a specific Protocol.

The internal management mode of each health facility allowed us to make a diagnosis on the issue later. Thanks to these data, the problem can be viewed in a general way and different solutions can be proposed.

The study's method is two-steps, which are:

1. Investigation by survey

2. Records of health establishments data collection

\section{Data collection \\ Sampling}

In our study, we considered that the main sources of MW are: Public and private hospitals, which are the largest producers of these kinds of waste.

Depending on the availability of facilities, the sample was restricted to 11 sources: 4 public hospitals, 6 private hospitals for-profit company and 1 private non-profit organization.

In order to gather the maximum data, we must have an idea about the local HWM. And we chose to act by participative surveys. Therefore, we had to put in place a survey for the qualitative data and another one for the quantitative data.

\section{Investigation by survey}

The survey, we have developed, deals with different aspects of HWM: waste production, storage, disposal, treatment, staffing, and training. We have also incorporated open-ended questions to get personal advice on the current situation.

This survey was designated to managers of health facilities, and /or hygiene technicians where these exist. In order to optimize the response rate, we chose to go to each of the concerned institutions, conduct a semi-directed survey and fill in the survey form.

If we had sent the survey to managers and let them answer it alone. The answers that could have been obtained would probably have been less specific and less faithful to reality.

All the interviews we conducted were accompanied by a visit to the concerned establishment premises. We were able to compare the given answers with the reality and discuss them with the nursing staff. The analysis of these answers gives an idea about the MWM quality and quantity. 
The knowledge of the mode of internal management of each health facility allowed us to make a diagnosis on the issue later. Thanks to these data, the problem may be considered in a comprehensive manner and different solutions can be proposed.

The method of study is a two-step, which:

1. Investigation by questionnaire

2. Records of health establishments data collection

\section{Results and discussion:-}

\section{Hospital waste management}

The answers obtained at the end of the questionnaire survey were treated by themes, distinguishing the situation of the different types of establishments. The percentages shown in the explanations represent the number of responses obtained.

\section{Quantities produced}

The amount of HW produced by the various health facilities in the city of Tangier is illustrates by Figure 2 . We find that this quantity varies from one institution to another.

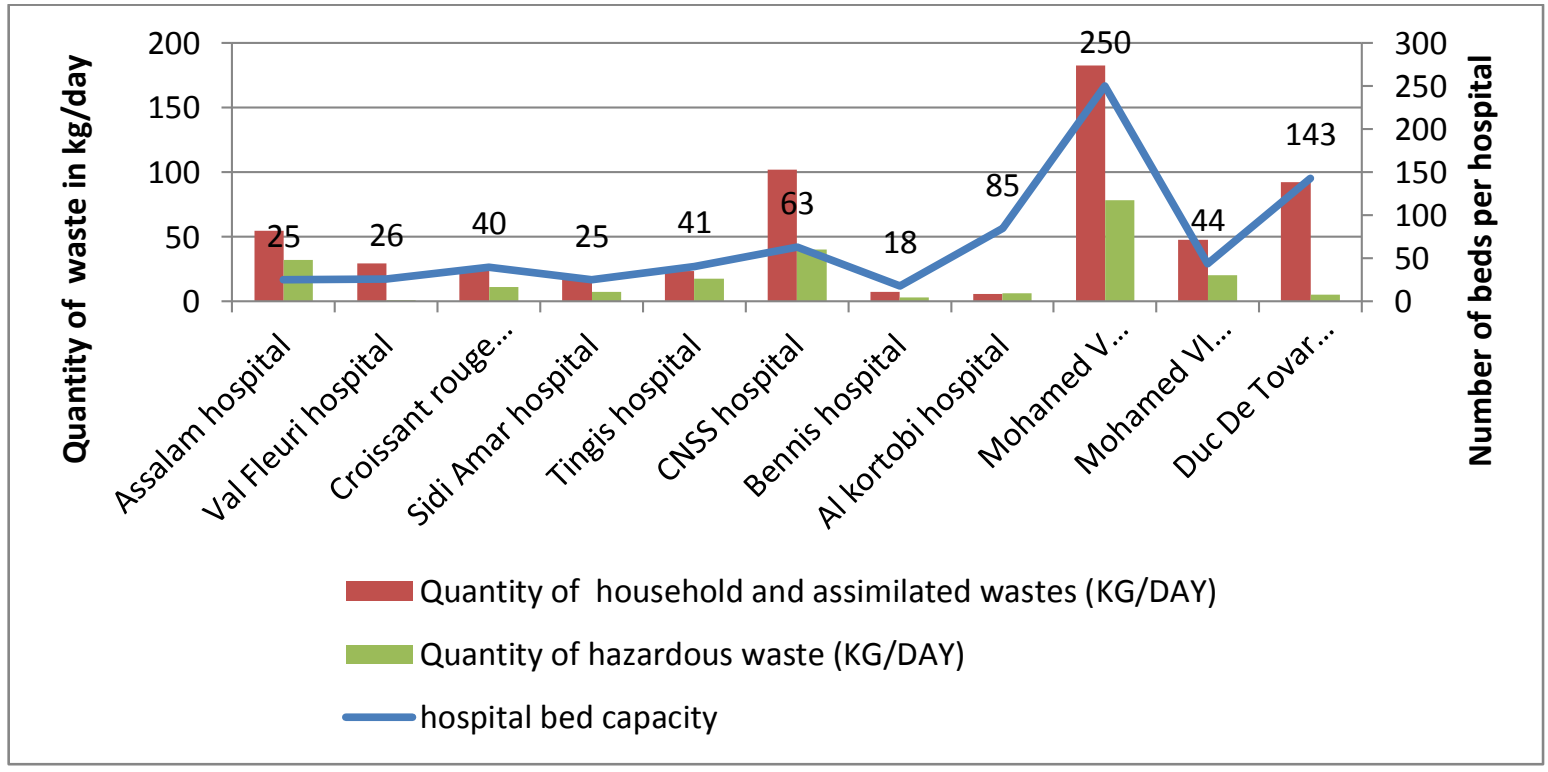

Figure 2:-Quantity of hospital waste produced by the different sanitary facilities in Tangier

By analyzing the histogram of weightings, it is found that the quantity of HW produced varies according to the litter capacity of the establishment; the more it is important the more the quantity of waste produced is high.

However, from Figure 3, we find that the amount of HSW varies between 2.18 and $0.07 \mathrm{~kg} / \mathrm{bed} / \mathrm{day}$ with an average of $0.89 \mathrm{~kg} / \mathrm{bed} /$ day. While the amount of waste from HCW varies between 1.29 and $0.03 \mathrm{~kg} / \mathrm{bed} / \mathrm{day}$ with an average of $0.37 \mathrm{~kg} / \mathrm{bed} /$ day. We also observe that we have three orders of dominance:

1. The quantity of HSW is much higher than that of HCW (case of the Val Fleuri Hospital and the Duc de Tovar Hospital)

2. The quantity of HSW is relatively higher than that of HCW (case of the majority of health establishments)

3. The quantity of HSW is similar to that of HCW (Case of AL KORTOBI hospital)

4. This distribution could be due to the sorting process in these establishments. 


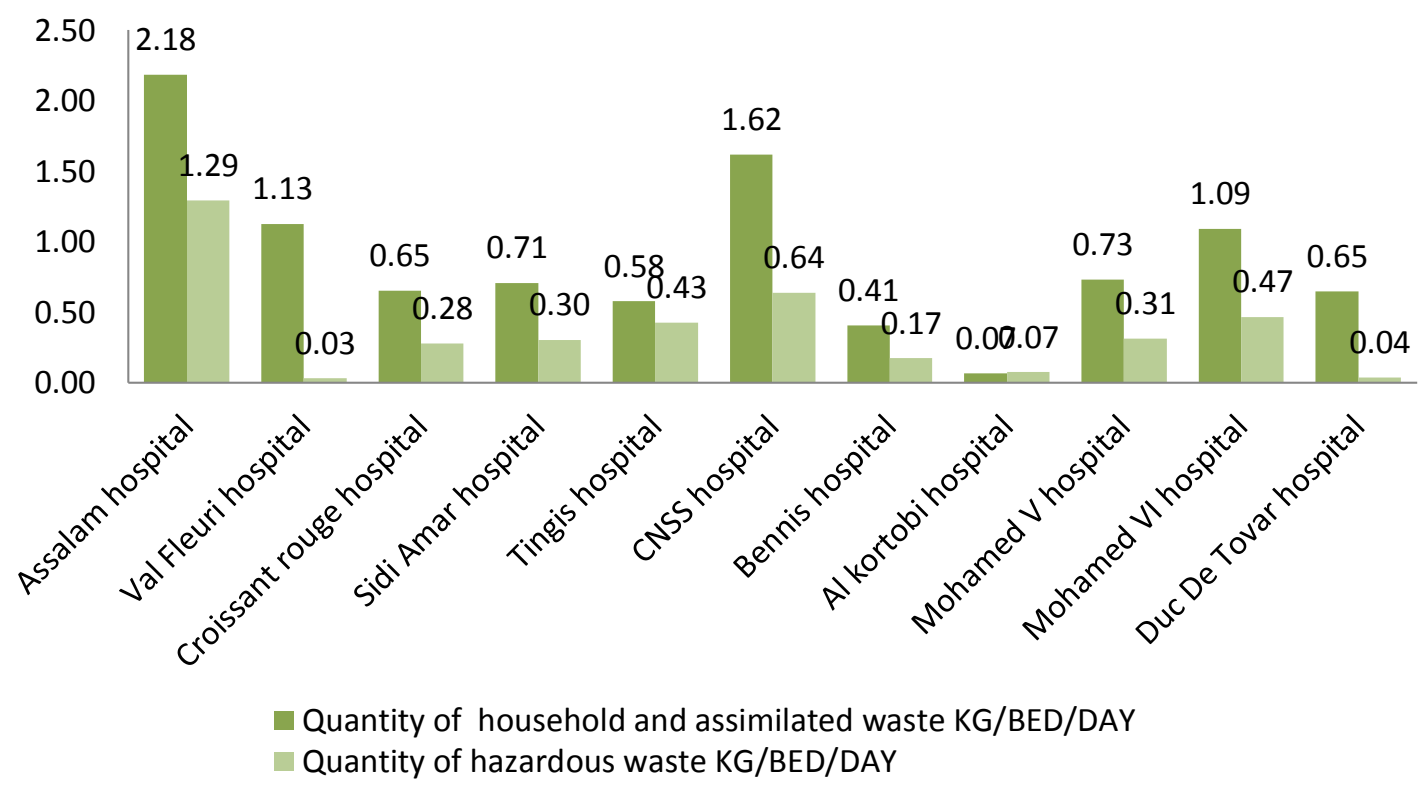

Figure 3:-Distribution of hospital waste in sanitary facilities in the city of Tangier ( $\mathrm{In} \mathrm{Kg} / \mathrm{bed} / \mathrm{day}$ )

The weekly weighing of hazardous waste is $81,25 \%$ of the studied establishments and it is carried out by the staff of the company ATHISA-Morocco, which is specialized in the collection and treatment of the HW. The solutions to be considered for the treatment of MW must, therefore, take this production into account.

One of our visits to one ofthe public hospitals of Tangier coincided with the day of the waste collection by theATHISA-Morocco staff. Thus we took advantage of this opportunity to attend the weighing, which was not very precise. The capacity of the scale, in this case, did not exceed $50 \mathrm{~kg}$; on the other hand, the containers weighed much more, especially those intended for the conservation of the placentas in the freezers until the day of the collection. In such a situation, the weighing personnel estimates the amount of waste when faced with such a problem.

For waste assimilated to household waste, they are collected daily by the services of the municipality or companies whose service has been delegated to them and are put directly in the landfill without any sorting.

\section{Waste sorting}

The sorting of hazardous and non-hazardous waste is the first step towards improving the MHW. This task makes it possible to envisage a suitable treatment thereafter. During the visits to the various services of the establishments studied, MW is mixed with household waste with the exception of two or three containers in each establishment. As for pungent objects, they are put directly into inviolable containers of yellow color reserved for these object after having hooded the bevels.

The interview with the professionals shows that the non-respect of the sorting is due sometimes to the lack of the material and the equipment: absence of red bags, sometimes stock-out of the black bags, adding to this the ignorance of this procedure by certain staff, whose behavior is difficult to change. However, this sorting is necessary for any clean waste management project.

\section{Sorting performing establishments}

Regarding the sorting between HCW and HSW, we find a significant difference between public and private institutions.

In fact, $84 \%$ of private establishments do not sort, mixing indifferently hazardous and non-hazardous waste in bins which are then sent to the public dump through a municipal collection. Only sorting Sharp Waste (SW) is done in 
almost $70 \%$ of cases. However, although collected separately, this SW is not subject to any specific treatment. It should be noted that sharp and SW can be immobilized or encapsulated in collection containers at the level of medical services. Thus, once filled at least their capacity, the containers will be filled with plaster and then transported to the landfill according to the testimony of a nurse from a private hospital. Regarding the public sector, all institutions have implemented sorting.

Again, the SW issue deserves separate treatment as $100 \%$ of public hospitals sort for this type of waste.

When we look at the quality of sorting done, we see a profound inefficiency. Some officials justify these deficiencies by the immobility of mentalities. However, by observing different services and treatment rooms, we realized that the problem comes from the nursing staff. Indeed, all waste produced in the room is thrown into the red bag regardless of their harmfulness. There is a strong presence of packaging, paper or leftover food in the middle of MW.

\section{Sorting by nursing staff:}

When the sorting is practiced in an establishment, it is generally the nursingstaffs who execute it. It is also the case that the maintenance staff takes in charge of waste sorting but this is a very rare case (9\% of establishments visited). The sorting of waste at the source is more efficient than waste sorting in another step. In the very rare cases where a control of the sort exists, it is the nurse's majors who assure it at the level of each service. These controls are never strict and do not result in penalties. They are only intended to identify black spots in sorting.

In terms of SW, the materials used are special yellow containers instead of cans or recovery bottles that have been seen in one of the private hospitals. However, SW must theoretically be fully collected in special, single-use containers, which leaves us to consider for the future large needs in special containers. This type of equipment is lacking in the private sector.Some private institutions have shown us stocks of this type of waste dating back several years.

On the other hand, ATHISA provides this material to public institutions. The quantities are sufficient and are not reused.

\section{Storage and disposal of waste}

The issues of storage and disposal of waste are extremely linked. In fact, storage times are a direct consequence of the frequency of collecting waste. For this reason, these elements will be treated together.

\section{Staff in charge of waste storage}

All health facilities have designated an employee who is responsible for waste storage. In $87.5 \%$ of the care establishments, maintenance staff takes care of it.

ATHISA agents institute $3 \%$ of cases, while the remaining $9.5 \%$ are cleaners who evacuate waste. Finally, they provide this function in all private hospitals.

\section{Storage equipment}

All the sanitary facilities visited, public and private ones, have a special room for storing waste. This room makes it possible to store the HCW and the HSW separately.

As for the private establishments that refused to welcome us, and according to the testimony of the neighborhood, they empty their waste directly into the HSW containers shared with the entire population of the neighborhood where they are located, despite the risks that may cause.

For the intermediate storage, all the studied establishments often use the corridors and the toilets, situation presenting a potential risk for people frequenting these places.

\section{Evacuation frequency}

All institutions surveyed, public and private ones evacuate their daily household and assimilated as well as MW already treated in situ. It is the municipal collection vehicle that takes care of this collection. In the case of external treatment of MW, the evacuation is done by ATHISA agents every week. 


\section{Treatment of waste from care activities}

All the establishments studied treat their risk waste in either situ or elsewhere.

\section{Integral waste treatment "in situ"}

Only one public institution has a sterilizer mill that processes about $400 \mathrm{~kg}$ of HSW every week.

The sterilizer crusher can treat all the waste produced by health establishments with the exception of blood products and placentas. Indeed, the coagulation of this waste with the action of heat causes a dysfunction of the blades of the mill and slows down, or even prevents the action thereof. It is therefore understood that establishments that will treat their HCW in situ will have to perform a very strict sorting of blood products so as not to hinder the action of the mill. As a result, they are sent elsewhere for specialized treatment.

Treatment of Health-care waste activities outside the institution All establishments studied, which do not have a sterilizer grinder, have their HCW treated outside. However, there are solutions for such treatment. Thus, these establishments eliminate all their waste in TETOUAN city by ATHISA, a company specialized in the treating MW. The technology used by ATHISA is the autoclaving which is based on sterilizing waste by destroying the microorganisms for a period of time by acting on a rise in temperature and pressure. These methods are those of the highest value of installation (3600 T/year).

\section{Personnel and training Internal management of waste}

After our investigation, only the public hospitals are equipped with a waste Manager, while we note his absence in all private hospitals under investigation.

However, it appears that, apart from hygiene technicians of Mohammed V hospital and CNSS polyclinic, none of the employees in question is trained specifically in waste management. Yet it is this employee responsible for the sector property from all the waste. So it must be able to set up and control the sorting, identification of equipment needed, to ensure the good knowledge of the waste management of the entire nursing staff and anything else conducive to proper management of waste.

In addition, this person, as well as any other employee having prolonged contact with the HCW, must have a reinforced medical follow-up. At present, this element is largely neglected. Indeed, while the follow-up of the nursing staff is generally satisfactory, the investigated staff is very largely insufficient or completely non-existent. It is, therefore, a question of establishing for this staff a frequent, regular and compulsory medical check-up.

\section{Training Nursing staff in waste sorting}

The results of the survey show that training the staff in waste sorting is substantially equivalent in both the public and private sectors. Thus, all the institutions declare having trained their staff the sorting protocol.

However, by about interviewing the various agents and caregivers, we found that some of them did not receive any training, as is the case with the Med VI hospital officer whose main job is security.
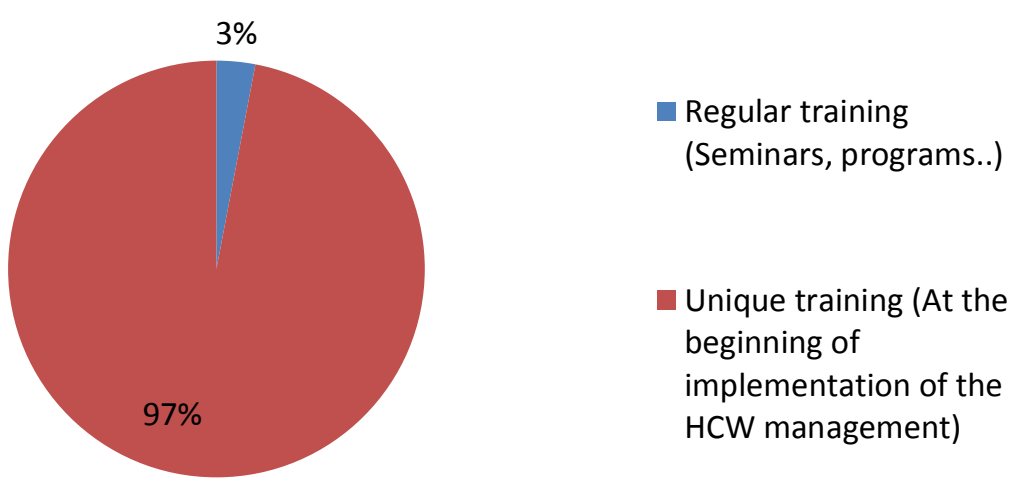

Figure 4:-Statistics of Training staff Waste Sorting 
We find that only $3 \%$ of the surveyed institutions provided ongoing training to their employees. In addition, it appears that a strong training need is felt by all interviewed actors who wish to set up a regular training program in both public and private sectors.

A particular attention should be paid to this training issue, because of its essential role in the proper hospital waste management. As part of the implementation of a well HCW management in Tangier city, it will be necessary to develop a comprehensive training program and ensure the staff good practices.

\section{General questions}

Deficiencies in waste management

In order to identify the actual needs of health actors regarding MW, the following question was asked: "In your opinion, what are the gaps in the current MWM process?". Therefore, several recurring problems can be highlighted as shown in the figure below.

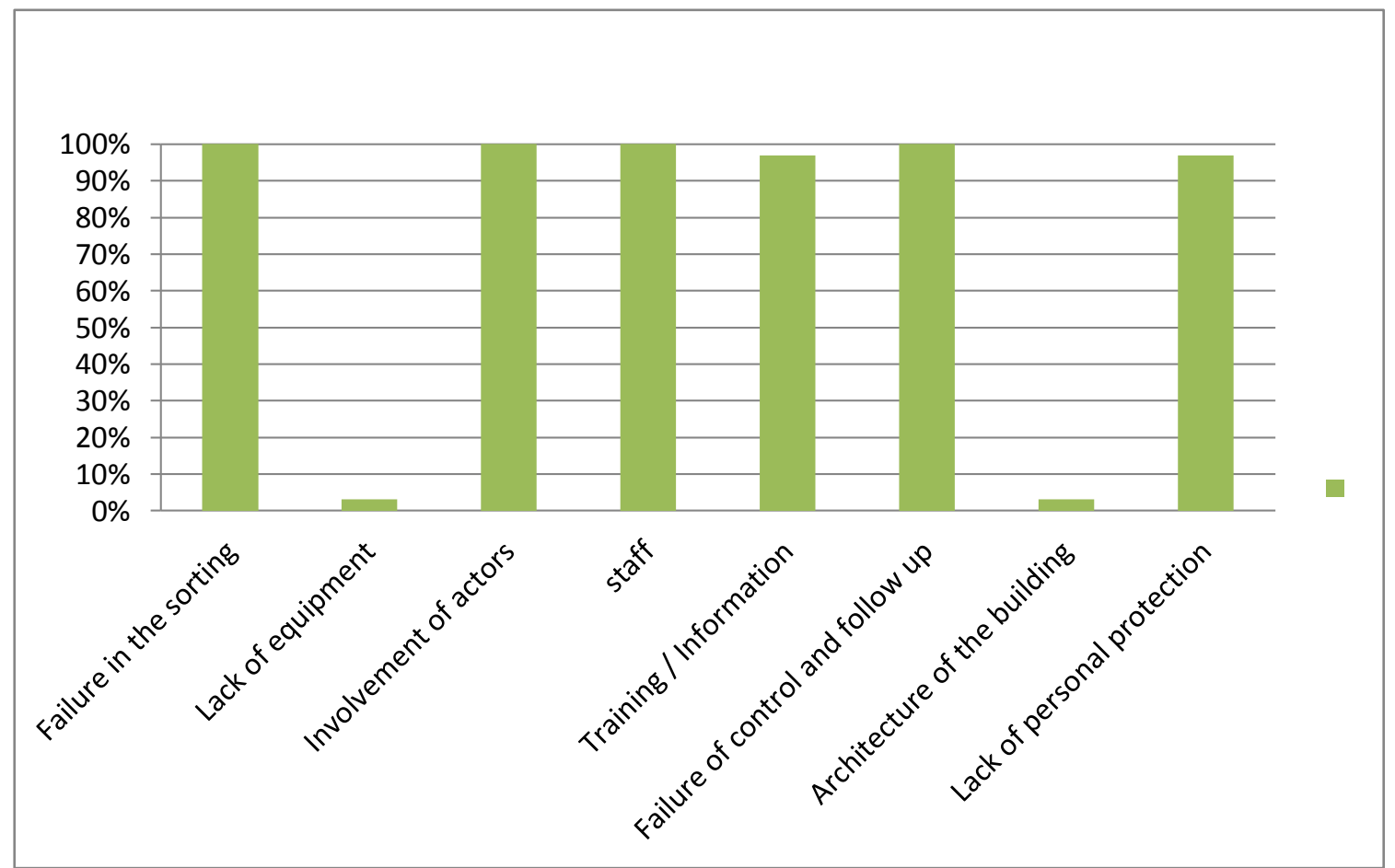

Figure 5:-Major shortcomings in the waste management process of sanitary facilities in Tangier city

According to Figure 5, we notice that health facilities in Tangierface the same problems, including:

1. Lack of training and information

2. An absence of control and monitoring

The main gap is currently observed, it concerns sorting risking and domestic waste as well as its establishment. Many private institutions are not performing waste sorting. Moreover, when sorting is performed, the quality is poor. No suitable treatment can work properly when these two types of waste are mixed. A substantive effort must, therefore, be made at all the city, in order to put in all places and establishments an efficient sorting.

\section{Conclusion and recommendations:-}

The diagnosis and inventory of waste management of health activities in Tangier city enabled us, through the analysis of available information in documents and sources, to put the finger on the need to set up a more rigorous management plan. We contested that:

1. There are no very reliable studies on the amount of HCW produced by public and private health care facilities in the city, which leads to a risk of overestimating quantities and oversizing treatment capacities. 
2. There is no sorting at the source carried out with rigor and according to well-elaborated protocols; this generates a major problem in the development of the management plans at the level of the care establishments.

3. The monitoring system (control and monitoring) specialized in waste management is absent.

4. The city and health care facilities do not have structures dedicated to the proper management of the various special waste streams (toxic, carcinogenic, etc.).

5. No hospital has a policy of recovery of recyclable materials. And in case there is a recycling channel, it remains at the informal stage.

To deal with this problem we recommend the following:

1. Recovery of recyclable materials must be included in the overall management of HSW and HCW in all health care facilities. Therefore, the need for preliminary studies on the nature of produced waste in each establishment to determinate their quantity, their typology and then programming the equipment for packaging, storage, transport and treatment, and finally the staff needed for these activities.

2. Training and sensitizing the health staff, hospital waste collection staff, patients and accompanying persons must be an integral part of the waste management plan since in many hospitals it has been found that human error is one of the most important causes of poor practice in the hospital waste management process.

3. Insufficient sorting and separation of waste were observed in all the establishments surveyed. For the most part, HSW has been mixed with IW. These were collected, transported and disposed of in the same way: landfill in the city's garbage dump.

4. For a better management of solid waste, there should be bins of different colors and special bags labeled well for infectious solid waste, and others for households.

5. Temporary waste storage sites should be kept clean to reduce spreading any disease.

\section{Références:-}

1. Alam, M.M., Sujauddin, M., Iqbal, G.M.A. and Huda, S.M.S. (2008) 'Report: Healthcare waste characterization in Chittagong Medical College Hospital, Bangladesh', Waste Management \&Research, Vol. 26, No. 3, pp.291296.

2. Ali, M. and Kuroiwa, C. (2009) 'Status and challenges of hospital solid waste management: case studies from Thailand, Pakistan, and Mongolia',

3. Almuneef, M. and Memish, Z.A. (2003) 'Effective medical waste management: it can be done', American Journal of Infection Control, Vol. 31, No. 3, pp.188-192.

4. Askarian, M., Vakili, M. and Kabir, G. (2004) 'Hospital waste management status in university hospitals of the Fars province, Iran', International Journal of Environmental Health Research, Vol. 14, No. 4, pp.295-305.

5. Chaerul, M., Tanaka, M. and Shekdar, A.V. (2008) 'A system dynamics approach for hospital waste management', Waste Management, Vol. 28, No. 2, pp.442-449.

6. Da Silva, C., Hoppe, A., Ravanello, M. and Mello, N. (2005) 'Medical wastes management in the south of Brazil', Waste Management, Vol. 25, No. 6, pp.600-605.

7. Esfandyari, Y., Mahdavi, Y., Seyedsalehi, M., Hoseini, M., Safari, G.H., Ghozikali, M.G., Kamani, H. and Jaafari, J. (2014) 'Degradation and biodegradability improvement of the olive mill wastewater by peroxielectrocoagulation/electrooxidation-electroflotation process with bipolar aluminum electrodes', Environmental Science and Pollution Research, Vol. 22, No. 8, pp.1-10.

8. Geng, Y., Ren, W-x., Xue, B., Fujita, T., Xi, F-m., Liu, Y. and Wang, M-1. (2013) 'Regional medical waste management in China: a case study of Shenyang', Journal of Material Cycles and Waste Management, Vol. 15, No. 3, pp.310-320.

9. Kamani, H., Hoseini, M., Seyed salehi, M., Mahdavi, Y., Jaafari, J. and Safari, G.H. (2014) 'Concentration and characterization of airborne particles in Tehran's subway system', Environmental Science and Pollution Research, Vol. 21, No. 12, pp.7319-7328.

10. Lee, B-K., Ellenbecker, M.J. and Moure-Eraso, R. (2002) 'Analyses of the recycling potential of medical plastic wastes', Waste Management, Vol. 22, No. 5, pp.461-470.

11. Liu, X. and $\mathrm{Yu}, \mathrm{D}$. (2003) 'Current status and future focus of hazardous waste management in China', Journal of Material Cycles and Waste Management, Vol. 5, No. 1, pp.0022-0025.

12. Nemathaga, F., Maringa, S. and Chimuka, L. (2008) 'Hospital solid waste management practices in Limpopo Province, South Africa: a case study of two hospitals', Waste Management, Vol. 28, No. 7, pp.1236-1245.

13. Prüss, A., Giroult, E. and Rushbrook, P. (1999) Safe Management of Wastes from Health-Care Activities, World Health Organization, Geneva. 
14. Tudor, T., Noonan, C. and Jenkin, L. (2005) 'Healthcare waste management: a case study from the National Health Service in Cornwall, United Kingdom', Waste Management, Vol. 25, No. 6, pp.606-615.

15. Tzanakos, K., Mimilidou, A., Anastasiadou, K., Stratakis, A. and Gidarakos, E. (2014) 'Solidification/stabilization of ash from medical waste incineration into geopolymers', Waste Management, Vol. 34, No. 10. 